\title{
Adsorption of Phenothiazines from Aqueous Solution by Aerosil ${ }^{1-3)}$

\author{
Naoki Nambu, Shotaro Sakurai, and Tsuneji Nagai \\ Hoshi Institute of Pharmacentical Sciences ${ }^{4}$
}

(Received August 1, 1973)

As a series of physico-chemical studies of an approach to an understanding of the membrane action of phenothiazines (PZS), adsorption of PZS from aqueous solution by carbon black, graphite, silica gel and polyethylene was already studied. ${ }^{5)}$

Aerosil, sometimes called white carbon and colloidal silica, is chemically a kind of anhydrous silicic acid, and has been widely used in the pharmaceutical field, for example, as glidants of powders and anti-sedimentary agent of suspensions. Moreover, it has such physical properties as the large specific surface area and very small apparent density, and its hydrophilic and hydrophobic balance is considered to depend on the ratio of hydrophilic moiety to hydrophobic moiety on the surface. Therefore, the interaction of drugs with Aerosil is interesting with a view to understanding of the adsorption phenomena of drugs on another kind of adsorbent than carbon black, in addition to getting some insight into the related pharmaceutical preparations.

\section{Experimental}

Materials_-Regarding the hydrophilic and hydrophobic balance of drugs, promazine, chlorpromazine, triflupromazine and chlorpromazine-sulfoxide (abbreviated as $\mathrm{PZ}, \mathrm{CPZ}, \mathrm{TPZ}$, and CPZ-O, respectively) were used as the samples.

Aerosil 200, 380, and R972 were supplied by Nippon Aerosil Co., Ltd. Aerosil 200 and 380 are of comparatively hydrophilic nature and have the specific surface areas of 200 and $380 \mathrm{~m}^{2} / \mathrm{g}$, respectively. Aerosil R972, which has been produced by methylating a part of silanol groups on the surface, is of comparatively hydrophobic nature.

Procedure for Determination of the Amount Adsorbed by Batch Method- $-70 \mathrm{mg}$ of adsorbent was added in $20 \mathrm{ml}$ of $1 / 30 \mathrm{M}$ phosphate buffer solution ( $\mathrm{pH} 7.00$, unless otherwise stated) of the respective drugs in a Nessler tube covered with aluminum foil at $30^{\circ}$ (unless otherwise stated), and then the procedure was carried out in the same way as already reported.5) The concentration of PZS was determined according to ultraviolet (UV) absorption method.5)

UV Absorption Spectroscopy by Reflection Method-UV absorption spectrum was obtained by reflection method using a Shimadzu 5000 spectrophotometer. The adsorbents were washed several times with water after the experiment of adsorption and dried under reduced-pressure, then UV spectrum was obtained by reflection method. Also the adsorbents before drying was subjected to the same UV spectroscopy.

\section{Result and Discussion}

\section{Adsorption Isotherms of Phenothiazines by Aerosil}

Examples of adsorption isotherms of PZS by Aerosil are shown in Fig. 1.

1) This paper forms Part XXVII of "Physico-chemical Approach to Biopharmaceutical Phenomena." Preceding paper, Part XXVI: K. Kono, Y. Takeda, H. Nogami, and T. Nagai, Chem. Pharm. Bull. (Tokyo), 22, 165 (1974).

2) A part of this work was presented at the 93rd Annual Meeting of the Pharmaceutical Society of Japan, Tokyo, April 1973.

3) "Aerosil" is the product name of Degussa Co., Ltd.

4) Location: Ebara-2-4-41, Shinagawa-ku, Tokyo, 142, Japan.

5) H. Nogami, T. Nagai, and N. Nambu, Chem. Pharm. Bull. (Tolkyo), 18, 1643 (1970). 


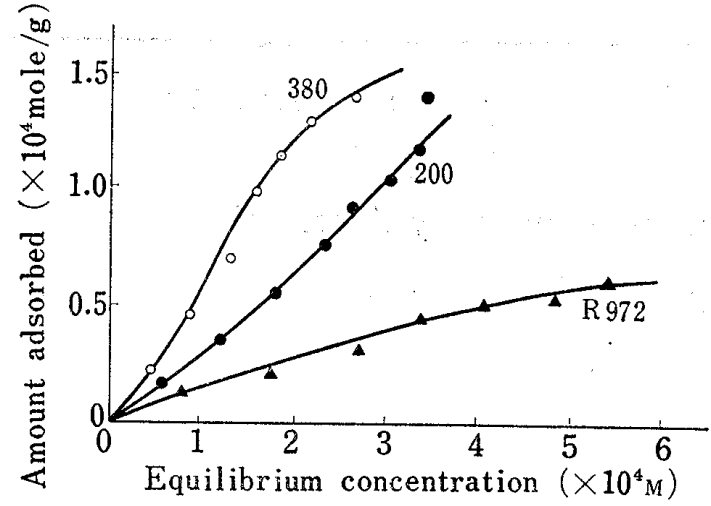

Fig. 1. Adsorption Isotherms of Chlorpromazine by Aerosil from $1 / 30 \mathrm{~m}$ Phosphate Buffer Solution ( $\mathrm{pH} 7.0)$ at $30^{\circ}$

Almost all the adsorption isotherms showed no saturation state following Langmuir's equation, as was similar to the case of polyethylene.5) This result suggested that the affinity of adsorbate to adsorbent was smaller than that of adsorbate to medium and also that the multi-layer adsorption of adsorbate or medium by physical bonding took place, as have been reported. ${ }^{6,7)}$ Therefore discussions were made on the basis of the amount adsorbed at constant equilibrium concentration, for example, at $3 \times 10^{-4} \mathrm{M}$ (Table I).

The amount of PZS adsorbed by Aerosil was of the order of $10^{-4}$ mole/g, while that at saturation by carbon black was of the order of $10^{-3}$ mole/g. This difference was very large compared with that in specific surface area between both adsorbents, suggesting that there was no strong binding between drug and silanol groups.

TABle I. Amount Adsorbed by Aerosil at Equilibrium Concentration $3 \times 10^{-4} \mathrm{M}\left(10^{4} \mathrm{~mole} / \mathrm{g}\right)$

\begin{tabular}{lccc}
\hline \multirow{2}{*}{ Drugs } & \multicolumn{2}{c}{ Adsorbents } \\
\cline { 3 - 4 } & Aerosil 200 & Aerosil 380 & Aerosil R972 $^{a)}$ \\
\hline Promazine & 1.50 & 0.94 & 0.14 \\
Chlorpromazine & 1.04 & 1.53 & 0.37 \\
Triflupromazine & 0.94 & 1.40 & 0.50 \\
Chlorpromazinesulfoxide & 0.83 & 0.96 & 0.02 \\
\hline
\end{tabular}

a) true density $2.2 \mathrm{~g} / \mathrm{cm}^{3}$, apparent density $0.06 \mathrm{~g} / \mathrm{cm}^{3}$

Among four PZS, adsorption isotherms of $\mathrm{CPZ}-\mathrm{O}$ were closely like to Langmuir type. This may suggest that $\mathrm{CPZ}-\mathrm{O}$ was so hydrophilic that multi-layer adsorption by weak bonding between CPZ-O molecules themselves did not take place.

\section{Adsorption of Phenothiazines in Relation to Hydrophilic and Hydrophobic Balance of Adsorbents and Drugs}

As shown in Table I, CPZ, TPZ and CPZ-O were more adsorbed by Aerosil 380 than 200 , but $\mathrm{PZ}$ was more adsorbed by 200 . There was found no clear relation between the specific surface area and the amount adsorbed, and the specific surface area did not form a predominant factor in the present adsorption. The amount adsorbed by Aerosil R.72 was smaller than those by 200 and 380 for each PZS. Aerosil R972 did not sediment in water and thus the adsorption is not the ordinary one at solid-liquid interface and the mechanism of adsorption is different from the other cases.

The order of hydrophobicity of drugs used is expected from their chemical structure as follows: $\mathrm{TPZ}>\mathrm{CPZ}>\mathrm{PZ}>\mathrm{CPZ}-\mathrm{O}$. As shown in Table $\mathrm{I}$, there was found no clear relation between the hydrophobicity and the hydrophilic and hydrophobic balance of drugs in the case of Aerosil 200 and 380. The amount adsorbed was not shown to be clearly related to the hydrophilic and hydrophobic balance of drugs. In the case of Aerosil R972, the larger the hydrophobicity of drugs is, the more increased the amount adsorbed.

6) T. Kagiya, Y. Sumida, and T. Tachi, Bull. Chem. Soc. Japan, 45, 1643 (1972).

7) L. Robert, C. R. Acad. Sci., Ser. C, 274, 309 (1972). 


\title{
Effect of Various Factors on the Adsorption
}

Using Aerosil 200, the lower the temperature was, the more increased the amount adsorbed of PZS. $\left(1.04 \times 10^{-4} \mathrm{~mole} / \mathrm{g}\right.$ at $30^{\circ}, 0.63 \times 10^{-4} \mathrm{~mole} / \mathrm{g}$ at $\left.40^{\circ}\right)$. Although the thermodynamic parameters of adsorption were not obtained, the result suggested that the adsorption of PZS by Aerosil might belong to physical adsorption type.

The fact that the amount adsorbed increased with $\mathrm{pH}$ may indicate that undissociated drug molecules are more liable to adsorb on Aerosil $\left(1.04 \times 10^{-4}\right.$ mole/g at $\mathrm{pH} 7.0,2.9 \times 10^{-5}$ mole/g at $\mathrm{pH} 6.0,1.5 \times 10^{-5} \mathrm{~mole} / \mathrm{g}$ at $\left.\mathrm{pH} 5.2\right)$, as was observed in the case of carbon black. ${ }^{5}$;

The more the concentration of sodium chloride and that of buffer solution increased, the more increased the amount adsorbed of PZS. This may indicate that water molecules around the surface of adsorbent are removed and drug molecules are adsorbed on those sites, as was observed in the case of carbon black and silica gel. ${ }^{\text {s) }}$

The more the concentration of urea increased, the more decreased the amount adsorbed $\left(1.04 \times 10^{-4} \mathrm{~mole} / \mathrm{g}\right.$ at urea free, $2.6 \times 10^{-5} \mathrm{~mole} / \mathrm{g}$ at $2 \mathrm{M}, 1.5 \times 10^{-5} \mathrm{~mole} / \mathrm{g}$ at $\left.4 \mathrm{~m}\right)$. This decrease in adsorption may be explained on the consideration that the adsorption of PZS proceeded on the hydrophobic interaction.5)

\section{UV Absorption Spectroscopy by Reflection Method}

UV absorption spectrum by reflection method of the adsorbent after the adsorption experiment gave a maximum at the same wave length as the case of drug only. The adsorbent without the adsorption experiment gave no maximal absorption. This result indicated that the drugs were adsorbed by Aerosil and it was considered possible that the adsorption of drugs by Aerosil may belong to the physical type.

Acknowledgement The authors gratefully acknowledge for the generous supports of the materials to Banyu Pharmaceutical Co., Ltd., Yoshitomi Pharmaceutical Co., Ltd. and Nippon Squibb Co., Ltd. Thanks are also given to Prof. Tominaga Keii's research group of Tokyo Institute of Technology for the performance of UV absorption spectroscopy by reflection method and to Miss Machiko Kubo for her assistance in the experimental work.

8) N. Nambu, T. Nagai, and H. Nogami, Chem. Pharm. Bull. (Tokyo), 19, 1058 (1971).

\section{Anomeric Proton Signals of the Component Monosaccharides of Free Oligoglycosides in Nuclear Magnetic Resonance Spectroscopy}

\author{
Kazumoto Miyahara and Toshio Kawasaki \\ Faculty of Pharmaceutical Sciences, Kyushu University ${ }^{\mathbf{1}}$
}

(Received October 16, 1973)

Nuclear magnetic resonance (NMR) spectroscopy has become an important means for structure elucidation in carbohydrate chemistry. A number of works have been reported on the determination of the glycosidic configurations and conformations of the component sugar units in oligoglycosides by use of the chemical shifts and the vicinal coupling constants

1) Location: Katakasu, 812, Fukuoka. 